\title{
Aspirin for Primary Prevention: What's a Clinician to Do?
}

\author{
Mitchell A. Psotka, MD, PhD ${ }^{7}$ and Kirsten E. Fleischmann, MD, MPH, FACC ${ }^{1,2}$ \\ 'Department of Medicine, Division of Cardiology, University of California San Francisco, San Francisco, CA, USA; ${ }^{2}$ UCSF School of Medicine, San \\ Francisco, CA, USA.
}

J Gen Intern Med 30(2):147-9

DOI: $10.1007 / \mathrm{s} 11606-014-3108-2$

(c) Society of General Internal Medicine 2014

$\mathrm{T}$ he United States Preventive Services Task Force (USPSTF) 2009 recommendations regarding the use of aspirin for primary prevention of cardiovascular disease (CVD) were intended to provide concise evidence-based management strategies. ${ }^{1}$ They endorsed the use of aspirin for primary prevention of myocardial infarction in men and for primary prevention of stroke in women when the benefits outweighed the potential harms. However, these guidelines were rapidly undermined by additional data, new reviews of previous trials, conflicting conclusions even among concurrent analyses, and varying thresholds for aspirin therapy among guidelines, professional societies, and other authoritative bodies. ${ }^{2}$

In this issue of JGIM, Fiscella and colleagues reveal that, based on patient report, the USPSTF recommendations are followed in relatively few patients. ${ }^{3}$ The authors analyzed National Health and Nutrition Examination Survey (NHANES) data from 2011 to 2012, 2 years after publication of the guidelines. Men between the ages of 45 and 79 years and women between 55 and 79 years, none of whom had known CVD, were included. To determine whether aspirin was merited by the published risk cutoffs, their 10 -year risk for CVD was calculated based on Framingham equations, as the USPSTF intended. ${ }^{1}$

Among those deemed eligible for aspirin therapy, only $34 \%$ of men and $42 \%$ of women reported that their physicians advised them to take it. ${ }^{3}$ In contrast, $76 \%$ of participants eligible for secondary prevention of CVD reported a physician recommendation for aspirin. Conversely, $24 \%$ of men and $28 \%$ of women who were not eligible for aspirin reported a physician recommendation to take it. USPSTF guidelinebased aspirin eligibility did not predict aspirin recommendation in multivariable logistic regression. Positive associations with older age, comorbidities such as diabetes mellitus, and access to healthcare suggest that traditional risk factors were employed for decision making, rather than the calculations and cutoffs recommended by the USPSTF.

The study is limited, most notably by relying on self-report, and by the fact that the authors did not have access to whether

Published online December 2, 2014 there were contraindications to aspirin therapy, such as a history of gastrointestinal bleeding. Given that hemorrhagic events are the predominant hazard that offsets the risk-benefit balance for aspirin use in primary prevention, this limitation is significant. Even so, that three-quarters of subjects eligible for secondary prevention recalled being advised to take aspirin suggests that neither failed report nor bleeding risk completely explain the low rates of aspirin recommendation reported in the primary prevention group.

So why are clinicians apparently not following the USPSTF guidelines fully? New data and analyses of the risks and benefits of aspirin therapy released shortly after the USPTSF guidelines may be partly responsible. The authors describe this changing landscape of published literature. The 2009 USPSTF guidelines had been published for only a few months when a novel individual participant data meta-analysis was published. ${ }^{4}$ This study demonstrated little if any benefit to aspirin for primary prevention, despite using data from the same six randomized controlled trials (RCTs) and over 90,000 subjects as prior investigations. This inquiry also debunked any improvement in stroke or a gender difference for primary prevention aspirin, tenets of the USPSTF publication.

Multiple publications over the subsequent years further altered the playing field. Three additional small RCTs and four meta-analyses were published before the end of $2012 .^{5}$ The results were disparate. While all found that aspirin decreased major cardiac events, they did not agree on whether it decreased CVD mortality, and those that evaluated adverse events found that there was modest net benefit, given a substantial increase in bleeding. If there was a benefit for aspirin as primary prevention, it was small, with a number needed to treat (NNT) of 120 over 6 years to avoid one CVD event, and 162 to avoid one nonfatal myocardial infarction (MI). ${ }^{5}$ The comparative number needed to harm (NNH) to cause one nontrivial bleeding event was 73 , which included fatal, cerebrovascular, retinal, or hollow viscous bleeding, any bleeding requiring hospitalization or transfusion, and bleeding termed "major" in the primary study.

Based on these investigations, the data for aspirin for primary prevention of CVD in 2011-2012 were murky at best, which was reflected in varying thresholds for treatment among authoritative guidelines. In contrast to the USPSTF, the American College of Chest Physicians (ACCP) recommended in 2012 that aspirin for persons over 50 years of age as primary prevention was a weak Grade $2 \mathrm{~B}$ recommendation. ${ }^{6}$ 
Additionally, the 2010 joint American Diabetes Association (ADA), American Heart Association (AHA), American College of Cardiology Foundation (ACCF) recommendations stated that aspirin for primary prevention was reasonable in patients with diabetes if their CVD risk was over $10 \%$ at 10 years and they did not have excess risk of adverse effects. ${ }^{7}$ This was a IIa recommendation even in patients considered to be near the highest end of the risk spectrum for CVD in the primary prevention population. So while all guidelines agree that individualized assessment of benefits and risks should guide aspirin therapy, the disparate strength of their recommendations and varying thresholds for treatment likely contribute to the relatively low rates of aspirin recommendation reported by Fiscella et al.

The difficulty in assessing individual bleeding risk continues to impair appropriate risk-benefit calculation. Most subjects in the completed primary prevention trials had less than a $2 \%$ predicted risk of CVD, and the increased bleeding with aspirin cancelled out much of the CVD benefit. It is presumed that at higher levels of CVD risk, the benefit of CVD protection will outweigh the risk. Nevertheless, bleeding risk is also higher in those with greater CVD risk, and currently too few trials have investigated this population to estimate the bleeding hazard accurately. ${ }^{5}$ In addition, various attributes that increase the propensity to bleed, such as a history of hemorrhage, have not been evaluated in a formal manner. Risk calculators as well as exclusions for certain comorbidities will likely be needed for appropriate aspirin implementation. Ongoing primary prevention clinical trials aim at higher risk subjects in an attempt to define the population that will benefit.

In 2014, the role for aspirin in primary prevention has been further muddied by emerging data on aspirin therapy for cancer prevention. No new meta-analyses regarding aspirin primary prevention for CVD have been published since 2012; however, at that time, some studies found decreased cancer mortality associated with aspirin administration, and a recent systematic review found that the effect on cancer mortality depended on which studies were included, with loss of benefit when more subjects were analyzed. ${ }^{8}$ This analysis again described the relatively modest absolute benefits and harms from aspirin therapy in the primary prevention population. For every 100,000 person-years of aspirin treatment, $60-84$ major CVD events, and possibly 34-36 colorectal cancer deaths, were avoided without a clear decrease in overall mortality (meta-analyses have reported mortality point estimates of less than one, but with confidence limits that include one). In contrast, for the same treatment period, aspirin would cause 99-178 nontrivial bleeds, including 8-10 hemorrhagic strokes and 68-117 gastrointestinal bleeds. As with aspirin for primary prevention of CVD, repeat analyses of the same data reveal conflicting conclusions for cancer prevention. ${ }^{8}$ Many ongoing trials have portions that address this role for aspirin and we will continue to watch this field closely.

In summary, the current state of aspirin recommendation for primary prevention of cardiovascular disease is complex. We know from the work of Fiscella and colleagues that aspirin is recommended by physicians for primary prevention of CVD in only a minority of USPSTF-eligible cases. Although nonfatal cardiovascular events are reduced, the totality of the evidence does not support a cardiovascular mortality benefit, and the potential role in cancer prevention remains incompletely supported even though it may one day supersede all CVD effects. So, what's a clinician to do? With no clear answers despite many meta-analyses, and guidelines with variable thresholds for treatment, we believe it is reasonable to individualize benefit and risk assessment and engage in shared decision making with our at-risk patients, much as has been advocated for initiation of statin therapy for primary prevention of CVD in patients with elevated risk. ${ }^{9}$ Our own practice is to consider aspirin for primary prevention for patients with a 10 -year risk of a first cardiovascular event surpassing $10 \%$, and for whom the benefit clearly exceeds the risk of bleeding, acknowledging that bleeding increases substantially with age or risk factors like prior hemorrhage, or NSAID or warfarin use. This discussion should ideally take into account patient preferences and values and the growing appreciation of the disutility associated with medication use for some people. ${ }^{10}$ However, given time constraints and the complex tradeoffs involved, we agree with Fiscella et al. that better tools to help busy clinicians assess the balance of benefit and risk would be helpful. Hopefully, ongoing trials will illuminate the situation further.

Acknowledgements: The authors would like to thank Myriam Hunink, Gabriel Gregoratos, Alan Venook and Allan Brett for their insightful comments.

Conflict of Interest: The authors declare that they do not have a conflict of interest.

Corresponding Author: Kirsten E. Fleischmann, MD, MPH, FACC; UCSF School of Medicine, Box 0124, 505 Parnassus Ave., San Francisco, CA 94143-0124, USA (e-mail: fleischm@medicine.ucsf.edu).

\section{REFERENCES}

1. United States Protective Services Task Force. Aspirin for the prevention of cardiovascular disease: U.S. Preventive Services Task Force recommendation statement. Ann Intern Med. 2009;150(6):396-404.

2. United States Department of Health and Human Services. Food and Drug Administration. Can an aspirin a day help prevent a heart attack? http:// www.fda.gov/downloads / forconsumers / consumerupdates / ucm395477.pdf (11/10/2014).

3. Fiscella K, Winters PC, Mendoza M, Noronha GJ, Swanger CM, Bisognano JD, Fortuna RJ. Do clinicians recommend aspirin to patients for primary prevention of cardiovascular disease? J Gen Intern Med. 2014. doi:10.1007/s11606-014-2985-8.

4. Antithrombotic Trialists $\mathbf{C}$, Baigent $\mathbf{C}$, Blackwell $\mathbf{L}$, Collins $\mathbf{R}$, Emberson J, Godwin J, Peto R, Buring J, Hennekens C, Kearney P, Meade T, Patrono C, Roncaglioni MC, Zanchetti A. Aspirin in the primary and secondary prevention of vascular disease: collaborative metaanalysis of individual participant data from randomised trials. Lancet. 2009;373(9678): 1849-60.

5. Seshasai SR, Wijesuriya S, Sivakumaran R, Nethercott S, Erqou S, Sattar N, Ray KK. Effect of aspirin on vascular and nonvascular 
outcomes: meta-analysis of randomized controlled trials. Arch Intern Med. 2012;172(3):209-16

6. Vandvik PO, Lincoff AM, Gore JM, Gutterman DD, Sonnenberg FA Alonso-Coello P, Akl EA, Lansberg MG, Guyatt GH, Spencer FA. American College of Chest P. Primary and secondary prevention of cardiovascular disease: Antithrombotic Therapy and Prevention of Thrombosis, 9th Ed: American College of Chest Physicians Evidence-Based Clinical Practice Guidelines. Chest. 2012;141(2 Suppl):e637S-68S.

7. Pignone M, Alberts MJ, Colwell JA, Cushman M, Inzucchi SE, Mukherjee D, Rosenson RS, Williams CD, Wilson PW, Kirkman MS. Aspirin for primary prevention of cardiovascular events in people with diabetes: a position statement of the American Diabetes Association, a scientific statement of the American Heart Association, and an expert consensus document of the American College of Cardiology Foundation. Circulation. 2010;121(24):2694-701.

8. Sutcliffe $\mathbf{P}$, Connock M, Gurung T, Freeman $\mathbf{K}$, Johnson S, NgiangaBakwin K, Grove A, Gurung B, Morrow S, Stranges S, Clarke A. Aspirin in primary prevention of cardiovascular disease and cancer: a systematic review of the balance of evidence from reviews of randomized trials. PLoS One. 2013;8(12):e81970.

9. Krumholz HM. The new cholesterol and blood pressure guidelines: perspective on the path forward. JAMA. 2014;311(14):1403-5.

10. Fontana M, Asaria P, Moraldo M, Finegold J, Hassanally $\mathbf{K}$, Manisty CH, Francis DP. Patient-accessible tool for shared decision making in cardiovascular primary prevention: balancing longevity benefits against medication disutility. Circulation. 2014;129(24):2539-46. 